\title{
Survival of midbrain dopamine neurons depends on the Bcl2 factor Mcl1
}

\author{
Edward J. Robinson', Sebastian P. Aguiar ${ }^{1}$, Willemieke M. Kouwenhoven', Dorinde S. Starmans ${ }^{1}$, Lars von Oerthel', \\ Marten P. Smidt (10 and Lars P. van der Heide ${ }^{1}$
}

\begin{abstract}
Mitochondria-dependent apoptosis plays an important role in the embryonic development of the midbrain dopaminergic system as well as in Parkinson's disease. Central to mitochondria-dependent apoptosis is the Bcl2 family of apoptosis-regulating proteins. However, it was unclear which $\mathrm{Bcl} 2$ proteins are important for the survival of dopaminergic neurons. Here, we identify Mcl1 as a critical Bcl2 pro-survival factor in midbrain dopaminergic neurons. Using a chemical biology approach to inhibit various components of the apoptotic machinery in the dopaminergic MN9D cell line or the control neuroblastoma N2A cell line, we find that functional inhibition of Mcl1 with the high affinity small molecule inhibitor UMI-77 results in a rapid and dose-dependent loss of viability, selectively in dopaminergic cells. In-depth analysis of the apoptotic signaling pathway reveals that chemical inhibition of Mcl1 results in the activation of Bax, activation of cleaved caspase- 3 and finally cell death. The dependence of mouse dopaminergic midbrain neurons on Mcl1 was confirmed using ex vivo slice cultures from Pitx3GFP/+ and wildtype mice. In mouse dopaminergic midbrain neurons positive for the midbrain dopaminergic marker Pitx3, or tyrosine hydroxylase, UMI-77 treatment caused a dramatic increase in cleaved caspase 3, indicating that Mcl1 activity is required for basal neuronal survival. Overall, our results suggest that Mcl1 is of critical importance to dopaminergic neurons and is a weak link in the chain controlling cellular survival. Boosting the pro-survival function of Mcl1 should be pursued as a therapeutic approach to augment the resilience of midbrain dopaminergic neurons to apoptotic stress in Parkinson's disease.
\end{abstract}

\section{Introduction}

Parkinson's disease (PD) is the second most common neurodegenerative disease after Alzheimer's disease ${ }^{1}$. Its clinical symptoms arise due to the progressive death of dopamine neurons in the substantia nigra pars compacta (SNpc) resulting in the loss of dopaminergic input to the striatum. There is no cure for Parkinson's disease and current palliative treatments are mostly based on supplementation with L-DOPA, the blood-brain-barrier permeable precursor for dopamine. At the onset of clinical symptoms it is estimated that more than $60 \%$ of the dopamine neurons have died ${ }^{1}$. Attenuating the loss of

Correspondence: Lars Heide (l.p.vanderheide@uva.nl)

'Swammerdam Institute for Life Sciences, University of Amsterdam, Science Park 904, 1098 XH Amsterdam, The Netherlands

Edited by R. Killick dopamine neurons would be a potential breakthrough in the treatment of PD. Of the different forms of cell death it is suggested that mitochondria-dependent apoptosis plays a major role in the loss of dopaminergic neurons in $\mathrm{PD}^{2,3}$, although the exact mechanism and signaling components remain obscure.

Mitochondria-dependent apoptosis is executed by the formation of a proteolipid pore in the outer mitochondrial membrane by oligomers of Bax and/or Bak ${ }^{4}$. The formation of these proteo-lipid pores allows components such as Cytochrome $C$, Apoptosis Inducing Factor (AIF), and Endonuclease $\mathrm{G}$ to leak out from the mitochondrial intermembrane space into the cytosol. Following mitochondrial outer membrane permeabilization (MOMP), mitochondrial contents spill into the cytosol to activate caspases resulting in the apoptotic phenotype ${ }^{4}$.

\section{(c) 2018 The Author(s)}

(c) (i) Open Access This article is licensed under a Creative Commons Attribution 4.0 International License, which permits use, sharing, adaptation, distribution and reproduction c. in any medium or format, as long as you give appropriate credit to the original author(s) and the source, provide a link to the Creative Commons license, and indicate if changes were made. The images or other third party material in this article are included in the article's Creative Commons license, unless indicated otherwise in a credit line to the material. If material is not included in the article's Creative Commons license and your intended use is not permitted by statutory regulation or exceeds the permitted use, you will need to obtain permission directly from the copyright holder. To view a copy of this license, visit http://creativecommons.org/licenses/by/4.0/. 
The apoptotic effector proteins Bax and Bak are the executioners of a precisely calibrated network of a large cast of pro and anti-apoptotic Bcl 2 family proteins ${ }^{4}$. The subfamily of anti-apoptotic $\mathrm{Bcl} 2$ proteins consists of $\mathrm{Bcl} 2$, Bcl-w, A1, Bcl-xl, and Mcl1. Whereas the pro-apoptotic Bcl2 family consists of the effector proteins Bax, Bak, and Bok and the BH3-only proteins, Noxa, Puma, Bim, Bad, Bid, Bmf, Hrk, Bik, and Bnip3.

We have previously suggested that development of the midbrain dopaminergic system depends on a complex interplay between factors controlling transcription, growth, and survival ${ }^{2}$. These factors control life and death of each individual dopamine neuron through the regulation of its unique set of $\mathrm{Bcl} 2$ factors. Recalibrating the $\mathrm{Bcl} 2$ composition in favor of cellular survival may strengthen dopaminergic neurons against stressful events which would normally result in apoptosis.

In this study, we set out to characterize the apoptotic machinery that controls dopaminergic cell death and identify the pro-survival Bcl2 family factor which is the weakest link for cellular survival. Previous data shows that overexpression of ectopic $\mathrm{Bcl} 2$ in dopamine neurons results in a higher number of dopamine neurons in mice after birth and protects against toxin-induced degeneration ${ }^{5}$. However, recent microarray experiments suggest that the relative expression levels of endogenous Mcl1 are higher in dopaminergic neurons as compared to $\mathrm{Bcl} 2^{6}$. Possibly, Mcl1 is of greater importance in mediating survival than $\mathrm{Bcl} 2$.

By leveraging the toolbox of high affinity small molecule inhibitors designed to induce apoptosis in cancer cells, we screened which inhibitors preferentially resulted in death of dopaminergic vs. non-dopaminergic neuronal cells. Our screen identified Mcl1 as crucial for dopaminergic neuronal survival, whereas $\mathrm{Bcl} 2$ and $\mathrm{Bcl}-\mathrm{xL}$ were of crucial importance in the non-dopaminergic neuronal cell line. In-depth analysis of the apoptotic pathway confirmed that Mcl1 indeed controls apoptosis via the canonical BaxCaspase-3 pathway. Additionally, overexpression of Mcl1 greatly augmented the resilience against the DNA-damage inducing apoptotic stressor etoposide in dopaminergic cells. Testing the resilience of Mcl1 in ex vivo mouse midbrain slices from both Pitx3GFP/+ and wildtype (WT) mice confirmed the importance of Mcl1 as a crucial $\mathrm{Bcl} 2$ factor in dopaminergic survival. Targeting the regulatory pathways controlling Mcl1 protein levels and function may therefore provide a novel therapeutic approach to strengthen dopaminergic neurons against apoptotic stress in Parkinson's disease.

\section{Results}

Mcl1 inhibitors preferentially induce caspase-3 activity in dopaminergic cells

Previous studies have suggested an important role for $\mathrm{Bcl} 2$ itself in controlling dopaminergic survival, whereas a role for Mcl1 has not been described ${ }^{5}$. However, microarray data ${ }^{6}$ and RT-qPCR data performed on sorted dopamine midbrain neurons from Pitx3GFP/+ heterozygous mice suggest that Mcl1 is one of the most highly expressed pro-survival $\mathrm{Bcl} 2$ factors in dopamine neurons. During mouse embryogenesis (not shown) as well as at P2, Mcl1 and Bcl-xL can abundantly be detected in dopaminergic neurons, whereas $B a x$ and $B c l 2$ are less present (Fig. 1a). To test the relative importance of $\mathrm{Bcl} 2$, Bcl-xL, and Mcl1, we used a small molecule chemical screen coupled with readouts for apoptotic cell death in two cell lines, the dopaminergic MN9D and the more commonly used non-dopaminergic neuroblastoma N2A cell line. The MN9D cell line was created by fusing microdissected E14 mouse rostral mesencephalic tegmentum cells from the approximate SNc/VTA with a neuroblastoma cell line (N18TG2) of neural crest origin. Unlike another commonly used DAergic cell line, PC12 (derived from an adrenal medullary tumor), MN9D dopamine content can be depleted by low dose MPP+, which is often used as a tool to induce PD like symptoms in rodents. MN9D cells are commonly used to test mechanisms and drug candidates relevant to PD. By contrasting the degree of cell death in MN9Ds vs. N2As, we aimed to identify weak links in survival that are specific to dopaminergic cells. To probe the relative weak links in apoptotic signaling of MN9Ds and N2As, the cells were dosed overnight with a panel of small molecule chemical inhibitors (Table 1, Fig. 1b), and their relative cell-type specificity was calculated (Fig. 1c). PAC1 is a caspase- 3 activator, and served as a positive control (while the signal was high, there was no significant difference between the cell lines). The small molecules ABT-263, ABT-199 preferentially induced caspase-3 activity in N2A cells as compared to MN9D cells which suggests a more prominent role for $\mathrm{Bcl} 2$ and $\mathrm{Bcl}-\mathrm{xL}$ as compared to Mcl1 ${ }^{10}$. High affinity inhibitors UMI-77, A1210477, and Obatoclax (which inhibit Mcl1 protein function) $)^{8}$, and YM155 (transcriptional inhibition of Mcl1 expression) preferentially induced caspase-3 activity in MN9D cells, which suggests that Mcl1 is of greater importance in dopaminergic cells as compared to N2A cells.

\section{Mcl1 but not $\mathrm{Bcl}-\mathrm{xL}$ is required for dopaminergic cell survival}

A time-curve experiment revealed that UMI-77 application to MN9D cells results in a significant increase in cleaved caspase-3 within 2-4 h (Fig. 2a). A dose-response curve revealed that at least $3 \mu \mathrm{M}$ of UMI-77 is enough to induce a significant increase in cleaved caspase-3 (Fig. 2b). To assess if the increase in cleaved caspase- 3 corresponds with the induction of cell death, MN9D cells were treated with propidium iodide (PI) after Mcl1 inhibition with UMI-77 (Fig. 2c). Live cells are impermeable to PI, 


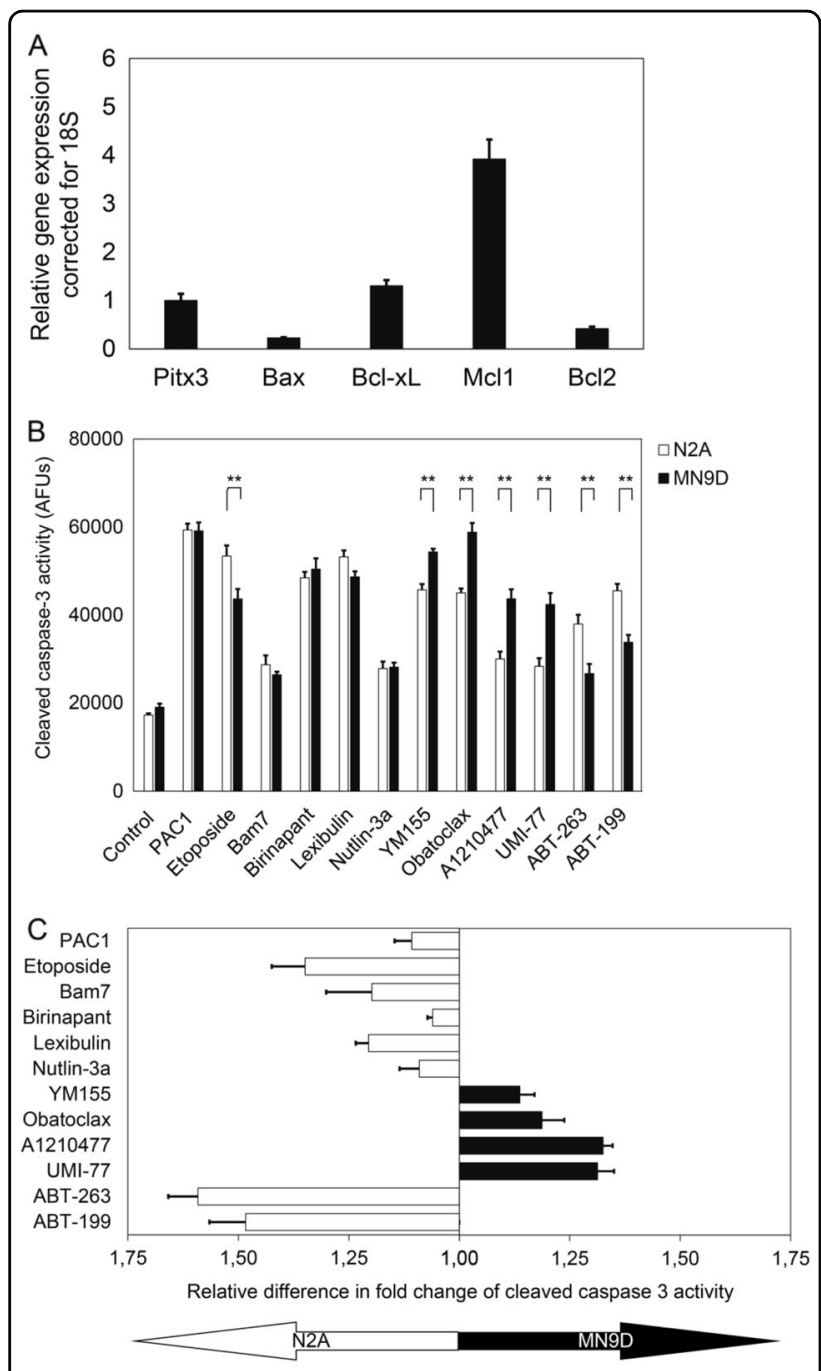

Fig. 1 a. Mcl1 is abundantly expressed in mouse midbrain dopaminergic neurons. 2-day old Pitx3/GFP mice were killed, brains were isolated, and dissociated with papain followed by FACsorting. GFP-positive cells were collected and RNA was purified before RTQPCR analysis with primers designed to specifically detect indicated transcripts. $\mathbf{b}$ Vulnerability of MN9D and N2A cell lines to a small molecule screen of apoptosis-inducing compounds. MN9D and N2A cells were incubated for $16 \mathrm{~h}$ at concentrations indicated. Cells were then treated with $0.1 \%$ Triton X-100 for $30 \mathrm{~min}$, followed by $30 \mathrm{~min}$ incubation in DEVD-AMC $(10 \mu \mathrm{M})$, a fluorogenic caspase 3 substrate. Fluorescence intensity was measured using a BioTek Synergy $\mathrm{H} 1$ plate reader. c Relative difference in fold change of cleaved caspase 3 activity of MN9D vs N2A cells. ( $n=3$, mean \pm SD. Unpaired Student's ttest, $p$-value: ${ }^{* *} P<0.01$ )

staining only the DNA of dying (late apoptotic) or dead cells. Indeed, the amount of PI-positive cells dramatically increased after UMI-77-dependent Mcl1 inhibition, confirming that UMI-77 induces cell death in MN9D cells (Fig. 2d).

Since UMI-77 had less effect on N2A cells as compared to MN9D cells (Fig. 1b, c), we compared several
Table 1 Small molecule inhibitors and stressors used in cleaved caspase 3 activity assay to determine the vulnerability of MN9D and N2A cells.

\begin{tabular}{lll} 
Compound & Works as & Concentration $(\boldsymbol{\mu M})$ \\
\hline PAC1 & Caspase-3 activator & 10 \\
Etoposide & Topoisomerase inhibitor & 5 \\
Bam7 & Bax activator & 10 \\
Birinapant & ClAP1 inhibitor /Birc2 inhibitor & 10 \\
Lexibulin & Microtubule inhibitor & 1 \\
Nutlin-3a & p53 inhibitor & 10 \\
YM155 & Mcl1 inhibitor /Survivin & 10 \\
& inhibitor & 10 \\
Obatoclax & Mcl1 inhibitor /Bcl2 inhibitor & 10 \\
A1210477 & Mcl1 inhibitor & 10 \\
UMI-77 & Mcl1 inhibitor & 10 \\
ABT-263 & Bcl-xL inhibitor & 10 \\
ABT-199 & Bcl2 inhibitor &
\end{tabular}

parameters between these cell types in response to UMI77 application. N2A cells did not show an increase in cleaved caspase-3 in response to UMI-77 application whereas MN9D cells did (Fig. 2e). Probing for Mcl1, the primary target of UMI-77, revealed that N2A cells express lower levels of Mcl1 as compared to MN9D cells, whereas $\mathrm{Bcl}-\mathrm{xL}$, the primary target of ABT- $263^{10}$, was expressed at comparable levels (Fig. 2e). Probing for Th confirmed the dopaminergic phenotype of MN9D cells and the nondopaminergic character of N2A cells as Th is not expressed in N2A cells. Th levels were not altered by UMI-77 incubation. To directly compare the effect of Mcl1 or Bcl-xL inhibition on cell death in N2A and MN9D cells, we incubated both cell types with either UMI-77, to inhibit Mcl1, or ABT-263, to inhibit Bcl-xL. UMI-77 induced a clear increase in cleaved caspase-3, but failed to do so in N2A cells (Fig. 2g, h). ABT-263 showed the inverse, as it did not result in a robust induction in cleaved caspase- 3 in MN9D cells, but did show a significant increase in N2A cell death, revealing cell typespecific effects and the reliance of MN9D cells on Mcl1 (Fig. 2g, h).

\section{Mcl1 prevents Bax-mediated canonical mitochondria- dependent apoptosis}

Inhibition of the anti-apoptotic function of Mcl1 results in an increase in cleaved caspase- 3 as well as an increase in the permeability of the cell to PI, strongly indicting a mitochondrial-dependent cell death pathway (Fig. 2). Canonical mitochondrial-dependent intrinsic apoptosis requires the activation of $\mathrm{Bax}$ and/or $\mathrm{Bak}^{4}$. Upon 

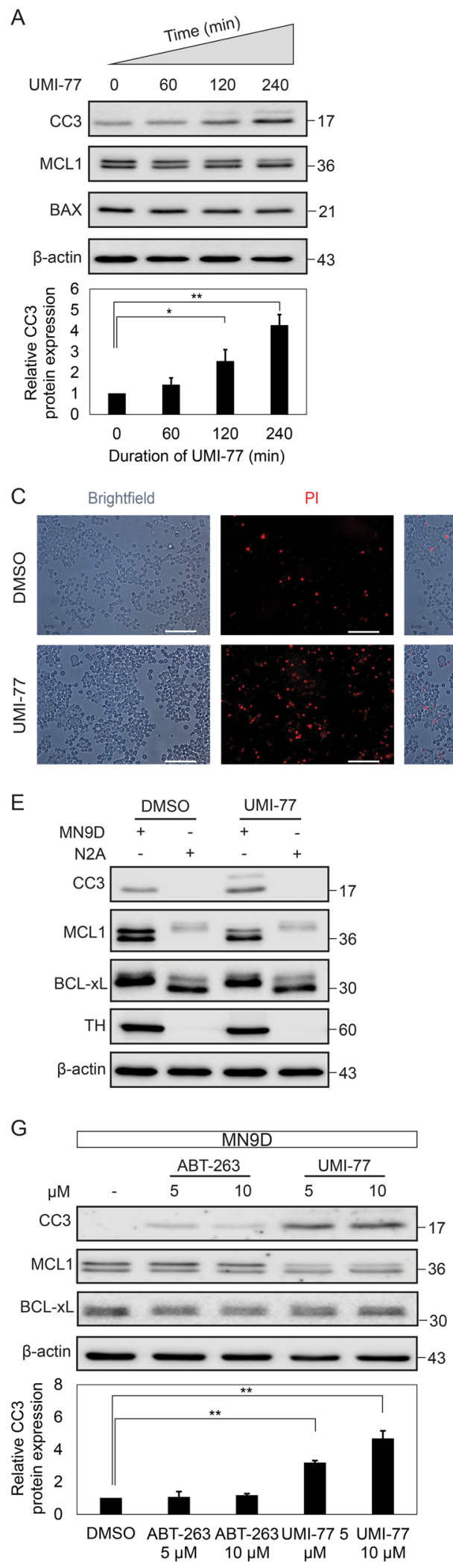

B

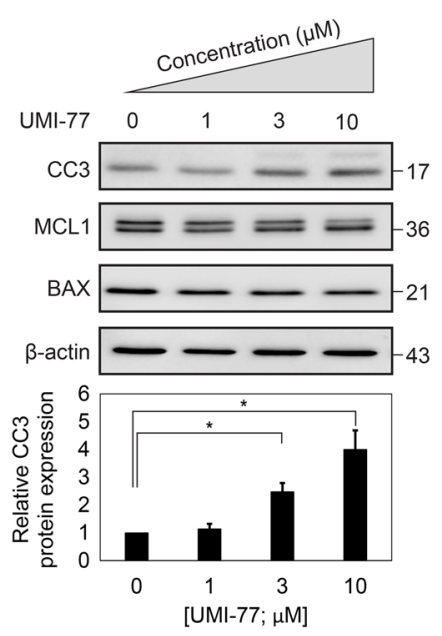

D

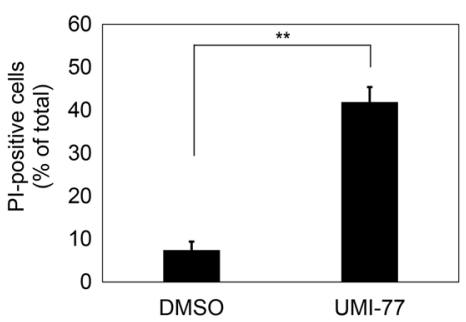

$\mathrm{F}$

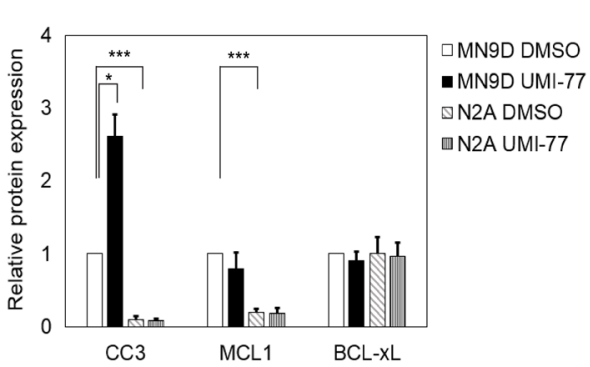

$\mathrm{H}$

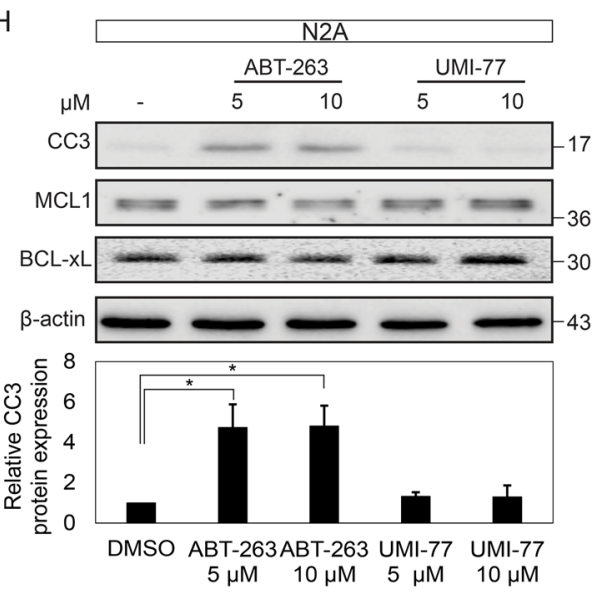

Fig. 2 (See legend on next page.) 
(see figure on previous page)

Fig. 2 Mcl1 inhibition leads to cell death of dopaminergic cells. a MN9D cells were grown and subsequently treated with $10 \mu \mathrm{M}$ UMI-77 for 60 , 120, or 240 min and subsequently analyzed by western blot for the amount of cleaved caspase 3. Mcl1, Bax, and actin were probed as controls. The amount of cleaved caspase 3 was normalized for the amount of actin and depicted in the bar graph. Quantification of the western blot shows that UMI-77 time-dependently increases the amount of cleaved caspase 3. b MN9D cells were grown as in $\mathbf{a}$, but treated with 1, 3, or 10 $\mu \mathrm{M}$ of UMI-77 before analysis on western blot and quantification. Again amount of cleaved caspase 3 was corrected for the amount of actin. c Cells were grown as in $\mathrm{A}$ and treated with $10 \mu \mathrm{M}$ of UMI-77 for $4 \mathrm{~h}$ and subsequently treated with propidium iodide to visualize permeant cells. Scale bar represents 50 $\mu \mathrm{m}$. $\mathbf{d}$ Positive cells were quantified and represented as relative amount compared to total amount of cells. e Dopaminergic MN9D cells and nondopaminergic cells were both treated with UMI-77 for $4 \mathrm{~h}$ and subsequently analyzed by western blot for the amount of cleaved caspase 3, Mcl1, Bcl$\mathrm{Xl}, \mathrm{Th}$, and actin. The actin corrected relative amount of cleaved caspase 3 is indicated in the bar graph. Additionally, levels of Mcl1 and Bcl1-xL were quantified and corrected for actin (f). g, h MN9D and N2A cells were treated with ABT-263 or UMI-77 at 5 and $10 \mu \mathrm{M}$ for $4 \mathrm{~h}$ and subsequently analyzed by western blotting for the amount of cleaved caspase $3, \mathrm{Mcl1}, \mathrm{BCl}-\mathrm{xL}$, and actin. Relative levels of cleaved caspase 3 were corrected for actin and depicted in the bar graphs. Significance was determined with a Student's -test, $p$ value: ${ }^{*} p<0.05,{ }^{* *} p<0.01,{ }^{* * *} p<0.005(n=3)$

activation, Bax undergoes a conformational change which exposes its $\mathrm{N}$-terminal domain. Visualization of this exposed domain with a conformation-specific epitope antibody revealed that its staining strongly correlated with activation of caspase 3 (Fig. 3a, b). Activated Bax and cleaved caspase 3 show a significant overlap and suggests a common pathway (Fig. 3c). The Bax6A7 positive, cleaved caspase 3 negative cells and the Bax6A7, cleaved caspase-3 positive cells may represent phases of cell death which are either too early or too late for both markers. Since canonical apoptosis depends on Bax activation followed by MOMP and subsequent caspase activation, inhibition of Bax activation should block apoptosis. BIP$\mathrm{V} 5$ is a 5-amino acid peptide designed to inhibit Bax activation. Pre-incubation of dopaminergic MN9D cells with BIP-V5 almost completely prevented the increase in cleaved caspase-3 upon the inhibition of Mcl1 with UMI77, confirming canonical mitochondrial-dependent apoptosis (Fig. 3e). Interestingly, the fact that the inhibition of Bax with a small peptide is able to prevent cell death supports the strategy that dopaminergic neurons can be strengthened by strategically boosting weak links in the pathways controlling survival - a finding in concordance with a previous report in which 6-OHDA induced nigral cell loss could be prevented by BIP-V $5^{11}$.

\section{Overexpression of $\mathrm{Mcl} 1$ protects against apoptotic stress}

Previously, it has been shown that ectopic overexpression of $\mathrm{Bcl} 2$ in dopaminergic neurons results in more dopaminergic neurons at birth as well as increased resistance to cell death-inducing stress ${ }^{5}$. Additionally, ectopic expression of the kinases SGK and AKT/PKB have been shown to exert a protective effect on dopamine neurons ${ }^{12,13}$. Interestingly, these kinases target various $\mathrm{Bcl} 2$ factors such as the BH3-only factor $\mathrm{Bad}^{14}$. More recently, the E3 ubiquitin ligase Parkin has been linked to the Bcl2 system, because Bax was described as one of the targets of Parkin, suggesting that mutations in Parkin would indirectly increase the levels of pro-apoptotic
$\mathrm{Bax}^{15}$. Since Mcl1 inhibition induces apoptosis more vigorously than the inhibition of other anti-apoptotic Bcl2 factors, we overexpressed Mcl1 together with GFP in MN9D cells and subsequently treated the cells with the apoptotic stressor etoposide (Fig. 4a). Quantification of PI- and GFP-positive cells reveal that under baseline conditions Mcl1 overexpression already reduces the frequency of cell death, although not significantly, suggesting pro-survival effects. Etoposide treatment dramatically increases the amount of PI positive cells in controls, whereas Mcl1 greatly attenuates the amount of dead cells, confirming its protective effects against apoptotic stressors (Fig. 4b).

\section{Mcl1 activity is required for survival of dopaminergic neurons ex vivo}

To expand our findings obtained in MN9D and N2A cells and to establish whether Mcl1 is of crucial importance for the survival of dopaminergic neurons in the mouse midbrain, we made use of ex vivo brain slice cultures using heterozygous Pitx3GFP $/+$ mice $^{9}$ These mice develop normally and express GFP in every dopaminergic midbrain neuron. Mice were killed and their brains were sliced into coronal sections. Left and right hemisphere were split. One hemisphere was treated with UMI-77 to inhibit Mcl1 function, whereas the counter hemisphere served as its control (Fig. 5a). After an overnight incubation, slices were fixed and stained with DAPI, as well as for GFP and cleaved caspase-3 (Fig. 5a). Using confocal microscopy, a representative part of substantia nigra was selected and images were quantified using semiautomated counting in ImageJ. Images were scored for the total amount of cells using DAPI, relative amount of GFP positive cells and relative amount of GFP and cleaved caspase-3 positive cells. After quantifying all DAPI positive nuclei, no significant reduction was observed between the control and UMI-77 treated slices $(n=7)$ (Fig. 5b). About $20 \%$ of the total amount of cells were GFP positive, again no difference was observed between conditions, 


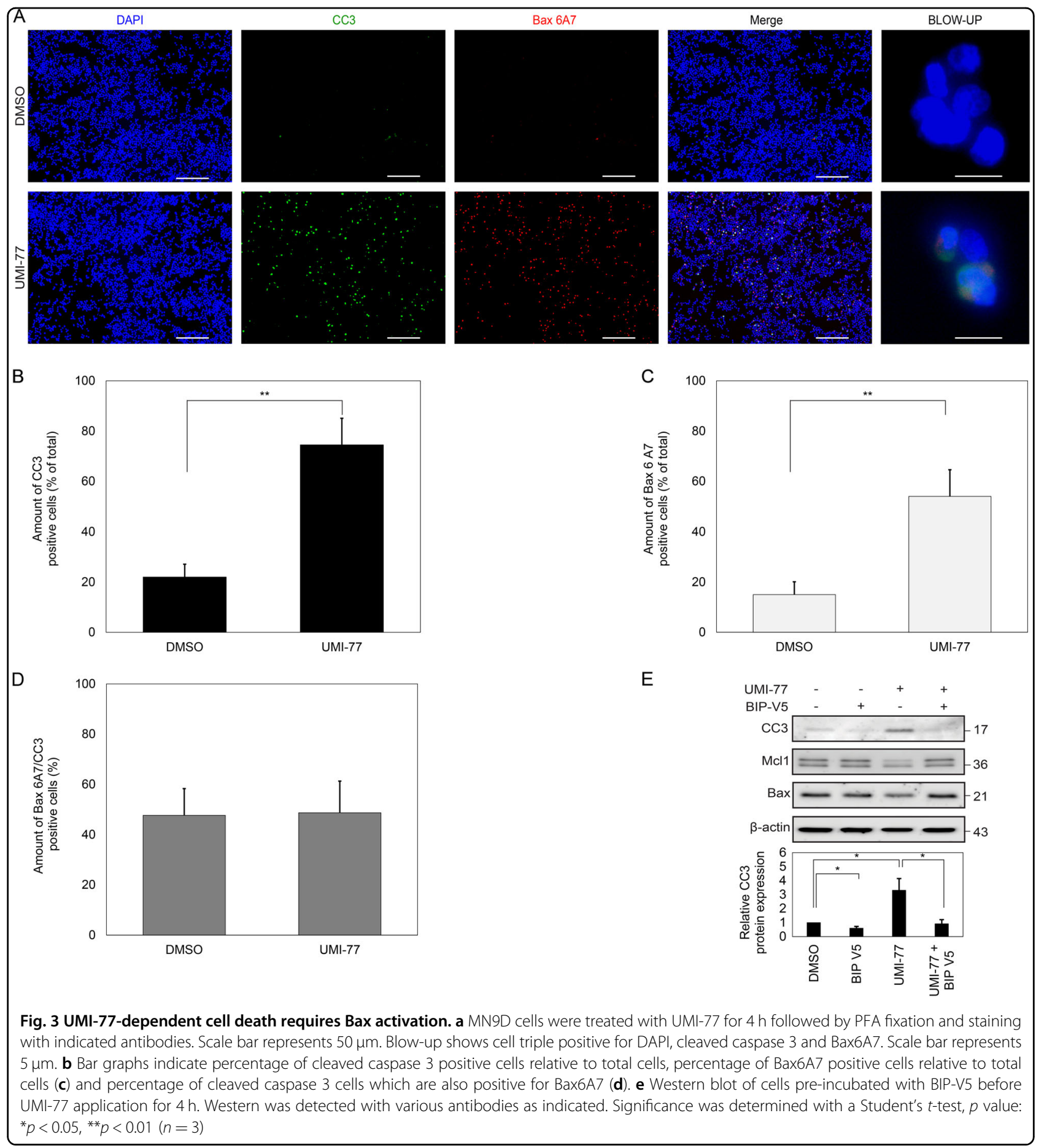

indicating that no obvious cell loss is observed after overnight treatment with UMI-77. Quantification of the number of GFP cells that were also positive for cleaved caspase- 3 showed that about 10 percent of the GFPpositive neurons were positive, suggesting that the slicing procedure and culture conditions are harsh for the intrinsically vulnerable dopaminergic neurons. Possibly, the elevated mitochondrial demands of dopaminergic neurons as well as the large size of their axonal arborization as compared to other neurons contribute to the high amount of basal cell death ${ }^{16}$. Quantification of the amount of cleaved caspase-3 positive cells after UMI-77 treatment shows a doubling of the amount of positive cells as compared to control $(n=7)$ (Fig. 5b). The increase in the amount of cleaved caspase 3 levels, supports an essential role for Mcl1 as an active survival factor in 


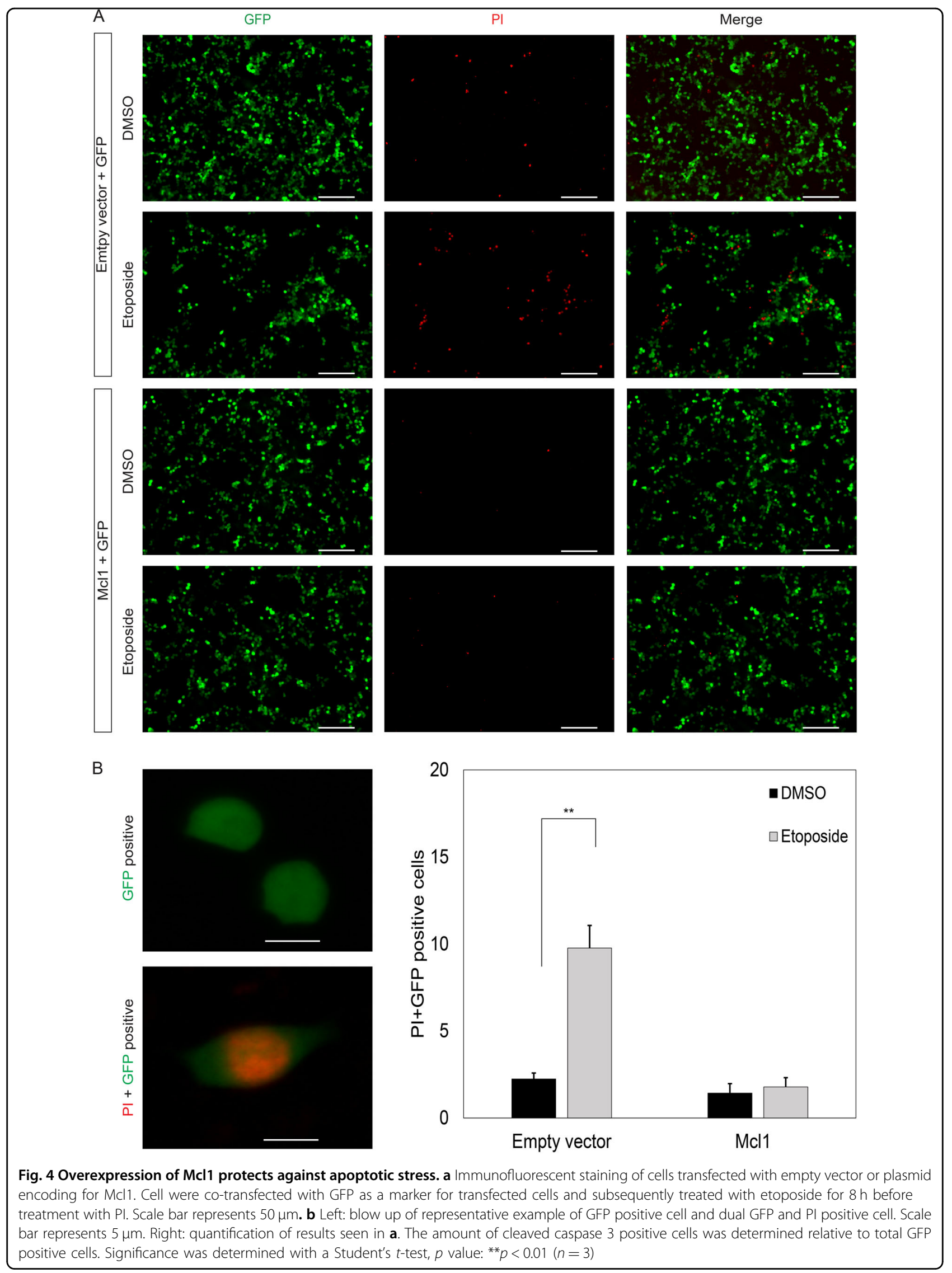




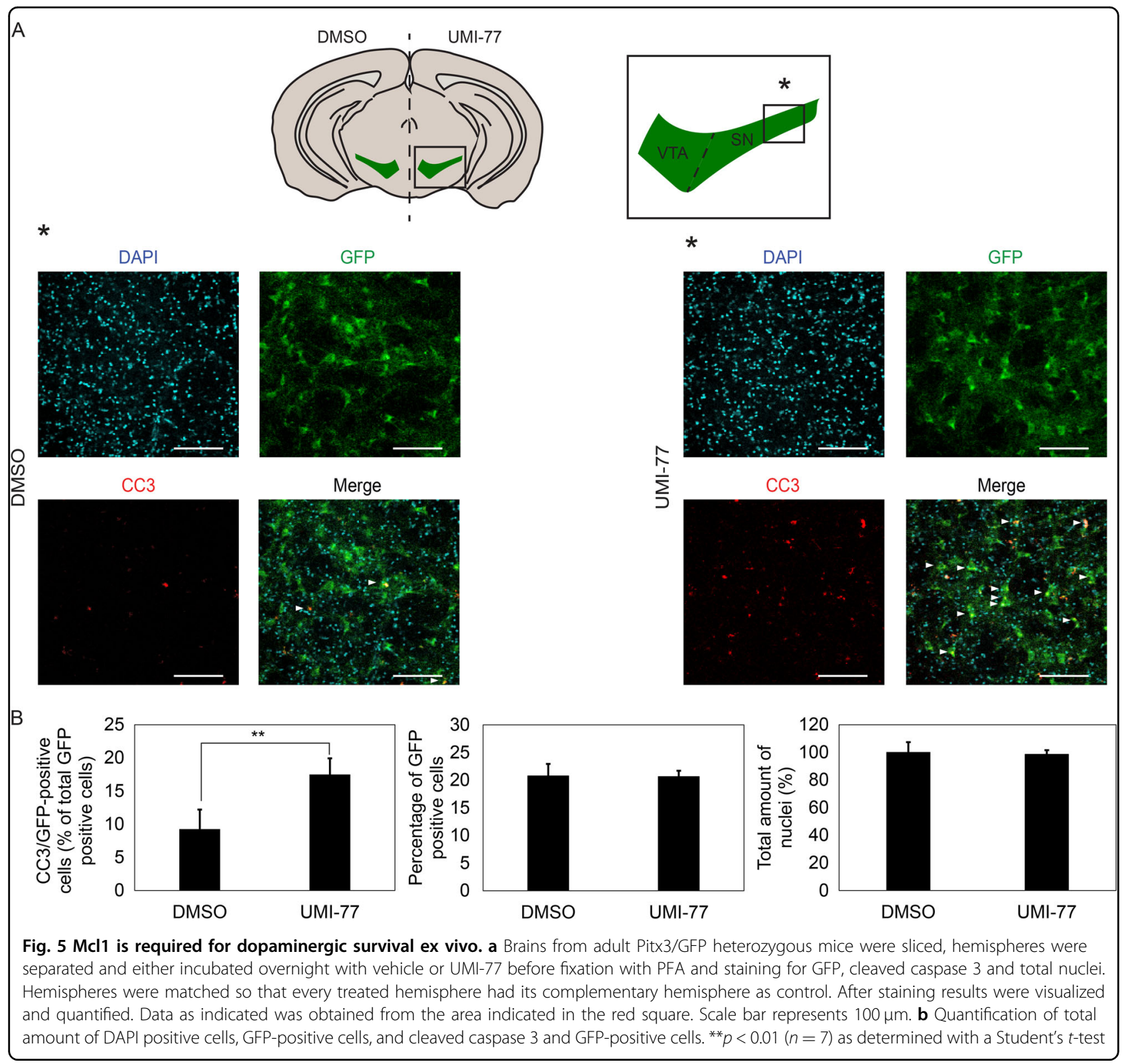

dopaminergic neurons. Since Pitx3GFP/ + heterozygous mice only have one copy of Pitx3 we repeated the experiment in mice containing Wt amounts of Pitx3 to exclude confounding effects caused by lower Pitx3 levels. Instead of GFP we stained Bl6 mice brain slices with Th as a marker for dopaminergic neurons (Fig. 6a). As for the Pitx3GFP/+ mice no significant reduction was observed in the total amount of DAPI positive cells (Fig. 6b). Quantification of the total amount of Th positive neurons indicates that there is also no loss after UMI-77 treatment as is observed for GFP in the Pitx3GFP/+mouse (Fig. 6b). Quantification of the amount of cleaved caspase 3positive Th positive neurons indicates that about 10 percent are positive for cleaved caspase 3. UMI-77 increases the amount of cleaved caspase 3 positive neurons to slightly under 20 percent (Fig. 6b). Results in both Pitx3GFP $/+\mathrm{P}$ as well as in Wt BL6 mice suggest that UMI-77 increases basal cleaved caspase 3 activity, under the apparently harsh culture conditions about two-fold. These results support the data obtained in vitro, suggesting Mcl1 is of crucial importance for the survival of dopaminergic neurons.

\section{Discussion}

Coordinated neuronal cell death in the mesencephalic dopaminergic system is crucial for proper embryonic 


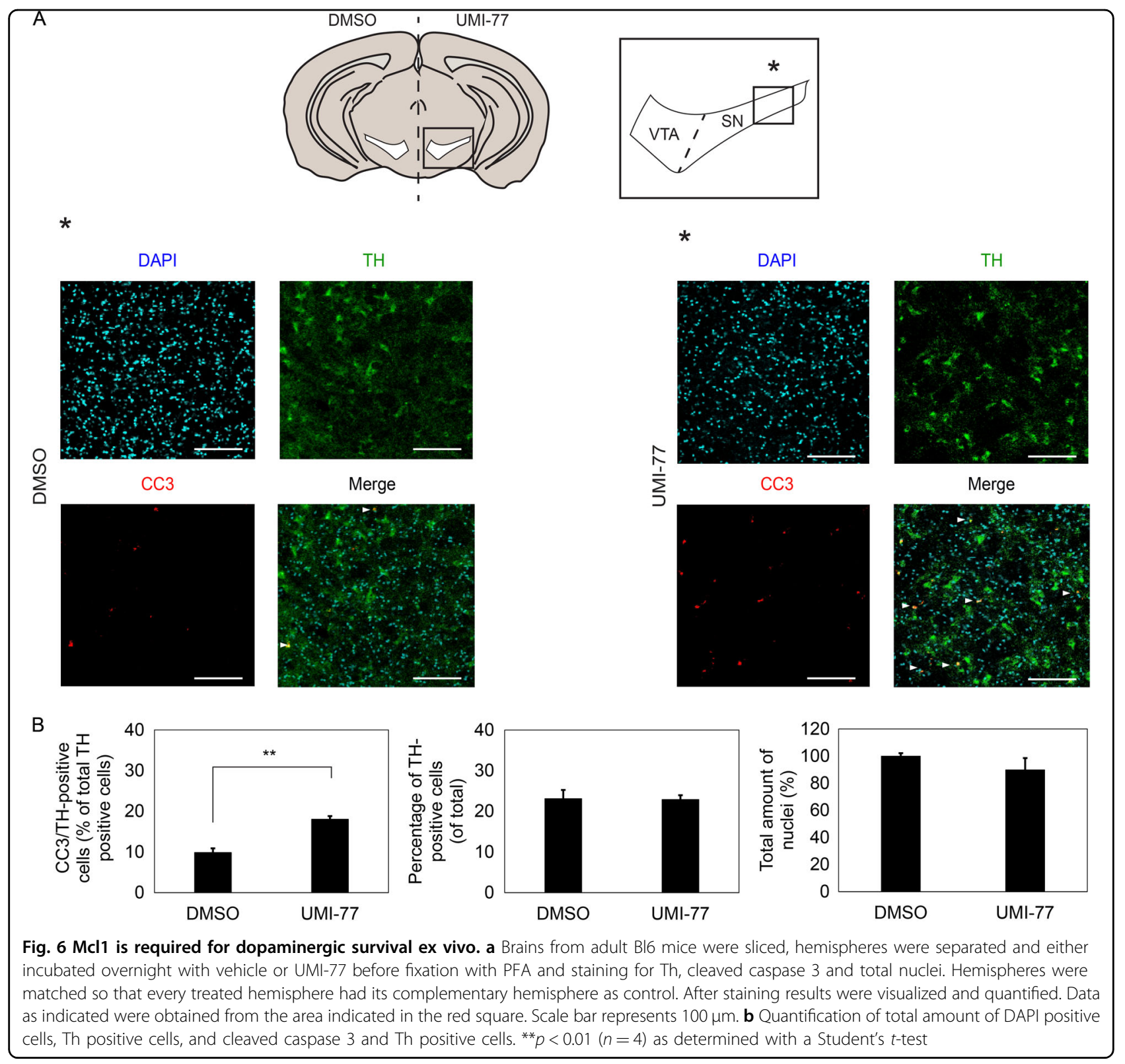

development, whereas aberrant cell death in this system with age is the ultimate cause for the clinical symptoms observed in $\mathrm{PD}^{1,2}$. Mechanistically, the form of cell death implicated in development and PD is mitochondrialdependent apoptosis which is controlled by various proand anti-apoptotic $\mathrm{Bcl} 2$ proteins $^{2,3}$. However, the crucial and most essential $\mathrm{Bcl} 2$ factors have not been identified.

Here, by comparing non-dopaminergic to dopaminergic cells, using an in vitro and ex vivo approach we have pinpointed the Bcl2 factor Mcl1 as a weak link in the system actively controlling neuronal survival and reveal a therapeutic target to enhance the resilience of this vulnerable population of cells.

\section{Mcl1 as a dopaminergic survival factor}

Historically, Mcl1 is the second identified pro-survival Bcl2 factor after Bcl2 itself ${ }^{17,18}$. The importance of Mcl1 during development is highlighted by the fact that knockout results in peri-implantation embryonic lethality ${ }^{19}$. Mcl1 is required for proper B- and T-cell maturation ${ }^{20}$, whereas various malignancies display elevated levels of $\mathrm{Mcl}^{21}$.

A handful of previous studies have suggested that various members of the $\mathrm{Bcl} 2$ family contribute to survival or cell death of dopaminergic neurons. For example, knockdown of Bax or Bak increases the viability of SHSY5Y dopaminergic cells in response to apoptotic stress, 


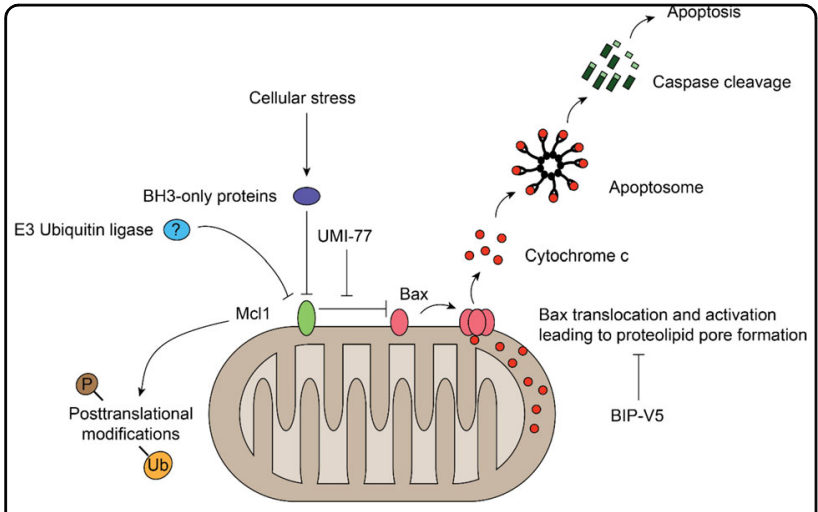

Fig. 7 Schematic model depicting the role of $\mathrm{Mcl} 1$ in dopaminergic survival. Mcl1 blocks the activation of Bax and thereby prevents mitochondrial outer membrane permeabilization, cytochrome $C$ release, and cleaved caspase 3 activation. Possibly regulation of Mcl1 by post-translational modifications can be exploited to therapeutically intervene with cell death occurring in dopaminergic neurons

whereas knockdown of Mcl1, Bcl2, or Bcl1-xL decreases viability $^{22}$. Transgenic ectopic expression of Bcl2 itself under the control of the Th promoter suggested that the $\mathrm{Bcl} 2$ family plays a positive role in controlling the number of dopaminergic neurons present after birth as well as determining the resilience towards apoptotic stressors ${ }^{5}$.

More recently, Mcl1 levels were shown to be altered in MPTP-treated mice. $24 \mathrm{~h}$ after the initial MPTP injections there was an expected marked reduction in dopamine terminals in the striatum as well as a drop in the amount of $\mathrm{Th}$ and dopamine transporter positive neurons. Whereas the amount of Bax remained statistically unchanged, the levels of Mcl1 were reduced by roughly $40 \%$. The authors suggest that Mcl1 degradation precedes Bax activation and the apoptotic phenotype ${ }^{23}$. Although suggestive, the functional contribution of Mcl1 as a protective dopaminergic cell survival factor was not investigated. Contrary to the previous study, MPTP has been shown to lead to an upregulation of Mcl1 at the messenger RNA and protein level in SH-SY5Y cells suggesting a compensatory mechanism ${ }^{24}$.

Most previous studies of the Bcl2 family in the nervous system used either knockdown strategies or overexpression techniques, possibly allowing secondary effects or compensation to occur. Since small molecule Bcl2 family inhibitors act rapidly at a specific binding site of the target and result in near complete blockade of a particular protein-protein interface, they are ideally suited to identify the most pivotal survival factors, circumventing the drawbacks of genetic techniques ${ }^{21}$. We employed a panel of small-molecule inhibitors of Bcl2 function developed by the pharmaceutical industry for use in oncology which allowed us to pinpoint Mcl1 as a key pro-survival $\mathrm{Bcl} 2$ factor in dopaminergic neurons. Importantly, the inhibitors which specifically interfere with the prosurvival function of Mcl1 had lesser effect on a nondopaminergic cell line, indicating that the inhibitors are not toxic to every cell-type, but display specificity (Figs. 1b, c and 2). Strikingly, UMI-77, a potent Mcl1 inhibitor (with an $\mathrm{IC}_{50}$ of $490 \mathrm{nM}$ ) rapidly and dosedependently induced the mitochondrial-dependent apoptotic pathway in MN9D cells, but not N2A cells (Fig. 2g). Western blotting indicates that N2A cell express low levels of Mcl1 as compared to MN9D cell, potentially explaining N2A's relative resistance to UMI-77 treatment (Fig. 2e). Contrary to Mcl1, the pro-survival Bcl2 factor Bcl-xL is comparably expressed in MN9D cells as well as N2A cells. Inhibition of Bcl-xL with ABT-263 resulted in apoptotic cell death of N2A, but spared MN9D cells.

The specificity of these small molecule Bcl2 inhibitors as well as the differential expression of $\mathrm{Bcl} 2$ factors in different cell types suggest that every cell type has a differently calibrated survival system ("Bcl2 code") which enables precise control of apoptosis during development, but reveals weak links with age and stress ${ }^{2}$. Intriguingly, molecular weights of Mcl1 as well as Bcl-xL appeared to differ between cell types suggesting that besides expression levels, post translational modifications of $\mathrm{Bcl} 2$ protein family members also have a cell type-specific signature (Fig. 2e).

\section{Targeting Mcl1 as a novel therapeutic approach in the treatment of PD?}

As discussed above, different cell types express different Bcl2 factors in addition to a cell-specific post-translational modification signature which could explain why dopaminergic neurons are more susceptible to stress in comparison to other cell types. The presence and importance of Mcl1 in dopaminergic neurons might be exploited to enhance the resilience of dopaminergic neurons to stress as is apparent from our described results. But what is the best strategy to enhance Mcl1 function? Mcl1 is a relatively short-lived protein, prone to various posttranslational modifications such as phosphorylation and ubiquitination ${ }^{14}$. Interfering with the degradation of Mcl1 may prove a promising strategy. Interestingly, the Bax E3 ligase Parkin has also been reported to target $\mathrm{Mcl1}^{22}$, implicating Parkin in controlling the balance between neuronal survival and cell death. How familial mutations in Parkin would shift the balance between this proapoptotic and pro-survival $\mathrm{Bcl} 2$ factor remains to be studied as well as if these results are the consequence of different cell types studied. More importantly, several additional E3 ubiquitin ligases have been identified that can tag Mcl1 for degradation, which includes Mcl-1 ubiquitin ligase E3 (Mule; also known as Huwe1 or ARFBP1), Trim17, beta transducin-containing protein ( $\beta$ TrCP), F-Box and WD containing 7 protein (FBW7), and 
cell-division cycle protein 20 activated anaphase promoting complex $\left(\mathrm{APC} / \mathrm{C}^{\mathrm{CDC} 20}\right)^{25-29}$. Conversely, ubiquitination of Mcl1 can be reversed by the deubiquitinase USP9X and the more recently identified USP $13^{30,31}$. The expanding list of potential Mcl1 E3 ligases emphasize the need to delve deeper into ubiquitination and degradation of Mcl1 in the dopamine system. Expression of any E3 ligase acting on Mcl1 specifically in the dopamine system would justify pursuing the development of small molecule inhibitors targeting this E3 ligase.

In conclusion, we have identified Mcl1 as a crucial Bcl2 factor essential for the survival of dopaminergic neurons. The mRNA and protein levels of Mcl1 as well as functional activity all contribute to dopaminergic resilience and may constitute the weakest link in the chain controlling dopaminergic survival (Fig. 7). Enhanced expression or functional protein activity of Mcl1 warrants a focused program of therapeutic development to attenuate dopamine neuron death in response to apoptotic stressors in Parkinson's disease. Further, the repertoire of $\mathrm{Bcl} 2$ factors ("Bcl2 code") and other apoptosis regulators appears to be uniquely balanced in different cell types beyond the CNS - opening the door to broader cell typespecific ablation or protection strategies.

\section{Material and methods \\ Animals}

All animal experimentation was supported and granted by the Animals Experimentation Committee of the University of Amsterdam according to national and international legislation. The morning of detection of the vaginal plug was considered as E0.5. Tissue was isolated at postnatal day P2 (day of birth +2 ). Animals are cared for on a daily basis according to rules and regulation of the Dutch and European law. Animals were killed according to rules and regulation of Dutch and European law.

\section{Fluorescence-activated cell sorting (FACS) and dissection}

Midbrains and rostral hindbrains of Pitx3GFP/+ heterozygous mice were dissected at P2 in L15-5\% Fetal Calf Serum (Gibco). Dissociation and sorting of midhindbrains were performed as described previously ${ }^{7}$. In short, freshly isolated tissue were dissociated using a Papain dissociation system (Worthington). Cells were sorted on a BD FACS Aria III using previously described settings $^{7}$ and collected in Trizol-LS (Invitrogen).

\section{Quantitative PCR}

Relative expression levels were determined by quantitative PCR (qPCR) real-time qPCR (Lightcycler 480) using the QuantiTect SYBR Green RT PCR Kit (QIAGEN) according to the manufacturer's instructions. For each reaction an estimated $0.1 \mathrm{ng}$ (FACsorted neurons) total RNA was used as input. Primer sequences used were:
Mcl1 forward 5'-CGTAACAAACTGGGGCAGGAT-3'; Mcl1 reverse 5'-CAAACCCATCCCAGCCTCTTT-3'; Bcl2 forward 5'-ACGGAGGCTGGGATGCCTTTG-3'; Bcl2 reverse 5 '-AGTGATGCAGGCCCCGACCA-3'; Bcl-xL forward 5'-CGGGGCACTGTGCGTGGAAA-3'; Bcl-xL reverse 5'-AAGTGTCCCAGCCGCCGTTC-3'; Bax forward 5'-TGGCAACTTCAACTGGGGCCG-3'; Bax reverse 5'-AGAGGAGGCCTTCCCAGCCAC-3'. Amplification products were separated on an agarose gel to confirm product size. Data was analyzed using the $\Delta \Delta \mathrm{Ct}$ method. Primers were assumed to have comparable efficiency.

\section{Chemical inhibitors and stressors}

UMI-77 (APExBIO); ${ }^{8}$ Bax-inhibiting peptide V5 (BIPV5; Sigma Aldrich); MG132 (Enzo Life Sciences), etoposide (Cell Signaling Technologies); PAC1, Bam7, Birinapant, Lexibulin (CYT997), Nutlin-3a, YM155, A1210477, Navitoclax (ABT-263), Venetoclax (ABT-199) and Obatoclax mesylate were all purchased from Selleckchem. All inhibitors and stressors were dissolved in DMSO; control conditions were all treated with the appropriate amount of DMSO.

\section{Cell culture and transfections}

MN9D cells were cultured on poly-D-lysine (SigmaAldrich) coated culture dishes in Dulbecco's Modified Eagle's Medium (DMEM; Lonza) supplemented with 200 nM L-glutamine (ThermoFisher Scientific), 1x Penicillin/ Streptomycin (Pen/Strep; 100 units/ml; ThermoFisher Scientific) and $10 \%$ heat inactivated fetal bovine serum (HiFBS; Biowest). Neuroblastoma 2 A cells were cultured in the same medium. Cells were incubated in a $5 \% \mathrm{CO}_{2} /$ $95 \% \mathrm{O}_{2}$ atmosphere at $37^{\circ} \mathrm{C}$. A day before the inhibitor and/or stressor experiments, cells were serum deprived in DMEM containing $200 \mathrm{nM}$ L-glutamine, $1 \mathrm{x}$ Pen/Strep and $0.5 \% \mathrm{HiFBS}$ to limit growth factor interference. Transfections were performed with lipofectamine 2000 (Invitrogen) according to manufacturer's instructions.

\section{Cleaved caspase-3 assay}

After treatment with the indicated inhibitors, cells were lysed with $0.1 \%$ Triton-X100 (Sigma-Aldrich) and incubated with $10 \mu \mathrm{M}$ AC-DEVD-AMC fluorogenic cleaved caspase-3 (CC3) substrate (ALX-260-031; Enzo Life Sciences) for $30 \mathrm{~min}$. Fluorescence was measured at an excitation wavelength of $350 \mathrm{~nm}$ and emission at $450 \mathrm{~nm}$ using a BioTek Synergy H1 plate reader.

\section{Western blotting}

After treatment, cells were washed with cold phosphate buffered saline (PBS; Lonza) and lysed in 1x Laemmli sample buffer (62.5 mM Tris-HCl pH 6.8, 2\% [SDS; Merck Millipore], $10 \%$ glycerol [Sigma-Aldrich] and 0.01\% w/v 
bromophenol blue [Sigma-Aldrich] in MilliQ water supplemented with $50 \mathrm{mM}$ dithiotretol [DTT; Merck Millipore]). Samples were collected, sonicated for $3 \mathrm{~min}$ in a Bioruptor sonicator (Diagenode) on HIGH settings and boiled at $95^{\circ} \mathrm{C}$ for $5 \mathrm{~min}$. Equal amounts of protein were loaded and separated on a SDS-PAGE gel (10-14\% depending on molecular weight), followed by transfer to Amersham Protran $0.2 \mu \mathrm{m}$ nitrocellulose membranes (GE Healthcare Life Sciences). Subsequently, membranes were blocked in 5\% ELK milkpowder (Campina) in Trisbuffered saline-0.1\% Tween-20 (TBS-T). Blots were incubated with the appropriate primary antibody in TBS$\mathrm{T}$ overnight at $4{ }^{\circ} \mathrm{C}$. Blots were incubated with goat-antirabbit Mcl1 (\#94296; 1:1000), goat-anti-rabbit Bcl-xL (\#2764; 1:1000), goat-anti-mouse $\beta$-actin (\#3700; 1:5000), goat-anti-rabbit Bax (\#14796; 1:1000), and goat-antirabbit CC3 (\#9664; 1:1000). All antibodies were purchased from Cell Signaling Technologies. After incubation with the appropriate HRP-conjugated secondary antibody (Invitrogen) in TBS-T, the blots were exposed to enhanced chemiluminescence (ECL) reagents. ECL reagents contained $250 \mathrm{mM}$ luminol (Fluka Analytical), $90 \mathrm{mM}$ p-coumaric acid (Sigma-Aldrich), $1 \mathrm{M}$ Tris- $\mathrm{HCl}$ $\mathrm{pH} 8.5$ and $0.02 \% \mathrm{H}_{2} \mathrm{O}_{2}$. Blots were detected on an Odyssey FC Imaging System (LI-COR Biosciences). Densitometric analyses were performed in ImageStudio Lite software (LI-COR Biosciences). Differences in signal between the conditions were tested for significance with a Student's $t$-test. Results were considered significant at $p<0.05$.

\section{Immunofluorescence \& propidium iodide staining}

For immunofluorescence experiments, MN9D cells were grown on poly-D-lysine coated coverslips. Cells were then serum deprived as described above and treated with the appropriate inhibitors/peptides. After treatment, cells were fixed for $10 \mathrm{~min}$ in $4 \%$ paraformaldehyde (PFA; Merck Millipore). Permeabilization was performed in $0.01 \%$ Triton X-100 in PBS for $30 \mathrm{~min}$, followed by PBS washes and blocking in 3\% bovine serum albumin (BSA; Sigma-Aldrich) and $0.01 \%$ Triton X-100 in PBS for $1 \mathrm{~h}$ at room temperature. Subsequently, cells were incubated overnight with goat-anti-mouse Bax 6A7 (Santa Cruz sc23959; 1:200) and goat-anti-rabbit CC3 (Cell Signaling Technologies \#9664; 1:200) at $4{ }^{\circ} \mathrm{C}$. After PBS washes, cells were incubated with goat-anti-mouse Alexa 555 and goat-anti-rabbit Alexa 488 secondary antibodies (ThermoFisher Scientific), respectively. Following PBS washes, nuclei were stained with DAPI (1:3000) and coverslips were embedded in Fluorsave Reagent (Merck Millipore). For propidium iodide (PI) experiments, cells were grown on poly-D-lysine coated coverslips in 12-wells plates, treated with the appropriate inhibitor, washed with cold PBS and immediately stained with PI (1:1000) for microscopic analysis. Images were obtained with a Leica DM5500 B microscope. The immunostained cells were analyzed using ImageJ software. Differences between conditions were tested for significance with a Student's $t$ test. Results were considered significant at $p<0.05$.

\section{Ex vivo slice culture \& free-floating immunohistochemistry}

Adult Pitx3GFP/ + heterozygous or wildtype mice (1 month old) brains were freshly isolated and sliced on a Leica VT100S vibratome in icecold PBS. Midbrain slices with a thickness of $250 \mu \mathrm{m}$ were obtained and checked for GFP expression in case of the Pitx3GFP/ + mice. Subsequently, the slices were divided in half to have an internal control. Slices were cultured overnight in explant culture medium as described previously ${ }^{9}$ in the presence of either DMSO or $10 \mu \mathrm{m}$ UMI-77 in a $5 \% \mathrm{CO}_{2} / 95 \% \mathrm{O}_{2}$ atmosphere at $37^{\circ} \mathrm{C}$. The next day, slices were briefly washed in PBS and fixed in $4 \%$ PFA for $30 \mathrm{~min}$ at $4{ }^{\circ} \mathrm{C}$. After fixation, the slices were briefly washed in PBS followed by blocking in $3 \%$ BSA, $0.5 \%$ Triton-X in PBS for $6 \mathrm{~h}$ at $4{ }^{\circ} \mathrm{C}$. Subsequently slices of Pitx3GFP/ + origin were incubated with goat-anti-chicken GFP (1:500; Abcam) and goat-antirabbit CC3 (1:200; CST) in PBS-T, whereas slices of wildtype mice were incubated with donkey-anti-sheep Th (1:500; Abcam) and goat-anti-rabbit CC3 (1:200; CST) in PBS-T overnight at $4{ }^{\circ} \mathrm{C}$. The next day, the slices were washed for 6 times $15 \mathrm{~min}$ in PBS-T and incubated with goat-anti-chicken Alexa 488 (1:500) and goat-anti-rabbit Alexa 555 (1:500) or alternatively with donkey-anti-sheep Alexa 488 (1:500) and goat-anti-rabbit Alexa 555 (1:500) for $4 \mathrm{~h}$ at RT. Following secondary antibody incubation, the slices were washed 4 times for $15 \mathrm{~min}$ and were incubated with DAPI (1:3000) to stain nuclei. Finally, slices were embedded on microscopy slides in Fluorsave reagent.

\section{Confocal microscopy}

Confocal images of the immunostained substantia nigra were obtained using a Nikon A1 confocal microscope. The following fluorphores were visualized with the following settings (excitation/emission): DAPI $405 \mathrm{~nm} /$ 425-475 nm), eGFP ( $488 \mathrm{~nm} / 500-550 \mathrm{~nm})$ and Alexa 594 $(594 \mathrm{~nm} / 570-620 \mathrm{~nm})$. Z-stack images were taken using a 20x objective. Additional settings: pinhole 0.7, scan size 1024 , speed 0.125 , and average 2 . Image analysis was performed using ImageJ software (Supplementary Fig. 1). Differences between conditions were tested for significance with a Student's $t$-test. Results were considered significant at $p<0.05$.

\footnotetext{
Acknowledgements

We would like to thank the LCAM center for technical assistance with the confocal microscopy. This work is supported by Stichting Parkinson Fonds.
} 


\section{Compliance with ethical standards}

\section{Conflict of interest}

The authors declare that they have no conflict of interest.

\section{Publisher's note}

Springer Nature remains neutral with regard to jurisdictional claims in published maps and institutional affiliations.

The online version of this article (https://doi.org/10.1038/s41420-018-0125-7) contains supplementary material, which is available to authorized users.

Received: 9 October 2018 Accepted: 16 October 2018

Published online: 21 November 2018

\section{References}

1. Dauer, W. \& Przedborski, S. Parkinson's disease: mechanisms and models. Neuron 39, 889-909 (2003).

2. van der Heide, L. P. \& Smidt, M. P. The BCL2 code to dopaminergic development and Parkinson's disease. Trends Mol. Med. 19, 211-216 (2013).

3. Venderova K., Park D. S. Programmed cell death in Parkinson's disease. Cold Spring Harb Perspect Med. 2, a009365 (2012).

4. Chipuk, J. E., Moldoveanu, T., Llambi, F., Parsons, M. J. \& Green, D. R. The BCL-2 family reunion. Mol. Cell 37, 299-310 (2010).

5. Jackson-Lewis, $\mathrm{V}$. et al. Developmental cell death in dopaminergic neurons of the substantia nigra of mice. J. Comp. Neurol. 424, 476-488 (2000).

6. Chakrabarty, K. et al. Genome wide expression profiling of the mesodiencephalic region identifies novel factors involved in early and late dopaminergic development. Biol. Open 1, 693-704 (2012).

7. Veenvliet, J. V. et al. Specification of dopaminergic subsets involves interplay of En1 and Pitx3. Development 140, 3373-3384 (2013).

8. Abulwerdi, F. et al. A novel small-molecule inhibitor of mcl-1 blocks pancreatic cancer growth in vitro and in vivo. Mol. Cancer Ther. 13, 565-575 (2014).

9. Jacobs, F. M. J. et al. Retinoic acid-dependent and -independent generegulatory pathways of Pitx3 in meso-diencephalic dopaminergic neurons. Development 138, 5213-5222 (2011).

10. Tse, C. et al. ABT-263: a potent and orally bioavailable BCl-2 family inhibitor. Cancer Res. 68, 3421-3428 (2008).

11. $\mathrm{Ma}, \mathrm{C}$. et al. Pre-administration of BAX-inhibiting peptides decrease the loss of the nigral dopaminergic neurons in rats. Life Sci. 144, 113-120 (2016).

12. Chen, $X$. et al. Neurotrophic effects of serum- and glucocorticoid-inducible kinase on adult murine mesencephalic dopamine neurons. J. Neurosci. J. Soc. Neurosci. 32, 11299-11308 (2012).

13. Ries, $V$. et al. Oncoprotein Akt/PKB induces trophic effects in murine models of Parkinson's disease. Proc. Natl Acad. Sci. USA 103, 18757-18762 (2006).
14. García-Sáez, A. J. The secrets of the Bcl-2 family. Cell Death Differ. 19 1733-1740 (2012).

15. Johnson, B. N., Berger, A. K., Cortese, G. P. \& Lavoie, M. J. The ubiquitin E3 ligase parkin regulates the proapoptotic function of Bax. Proc. Natl Acad. Sci. USA 109, 6283-6288 (2012).

16. Pacelli, C. et al. Elevated mitochondrial bioenergetics and axonal arborization size are key contributors to the vulnerability of dopamine neurons. Curr. Biol. 25, 2349-2360 (2015).

17. Kozopas, K. M., Yang, T., Buchan, H. L., Zhou, P. \& Craig, R. W. MCL1, a gene expressed in programmed myeloid cell differentiation, has sequence similarity to BCL2. Proc. Natl Acad. Sci. USA 90, 3516-3520 (1993).

18. Tsujimoto, Y., Cossman, J., Jaffe, E. \& Croce, C. M. Involvement of the bcl-2 gene in human follicular lymphoma. Science 228, 1440-1443 (1985).

19. Rinkenberger, J. L., Horning, S., Klocke, B., Roth, K. \& Korsmeyer, S. J. Mcl-1 deficiency results in peri-implantation embryonic lethality. Genes Dev. 14, 23-27 (2000).

20. Opferman, J. T. et al. Obligate role of anti-apoptotic MCL-1 in the survival of hematopoietic stem cells. Science 307, 1101-1104 (2005).

21. Belmar, J. \& Fesik, S. W. Small molecule Mcl-1 inhibitors for the treatment of cancer. Pharmacol. Ther. 145, 76-84 (2015).

22. Carroll, R. G., Hollville, E. \& Martin, S. J. Parkin sensitizes toward apoptosis induced by mitochondrial depolarization through promoting degradation of Mcl-1. Cell Rep. 9, 1538-1553 (2014).

23. Lu, E., Sarkar, S., Raymick, J., Paule, M. G. \& Gu, Q. Decreased Mcl-1 protein level in the striatum of 1-methyl-4-phenyl-1,2,3,6-tetrahydropyridine (MPTP)-treated mice. Brain Res. 1678, 432-439 (2018).

24. Fan, L., Jiang, L. \& Du, Z. Myeloid cell leukemia 1 (Mcl(-1)) protects against 1methyl-4-phenylpyridinium ion (MPP+) induced apoptosis in Parkinson's disease. Metab. Brain Dis. 30, 1269-1274 (2015).

25. Ding, Q. et al. Degradation of Mcl-1 by beta-TrCP mediates glycogen synthase kinase 3-induced tumor suppression and chemosensitization. Mol. Cell. Biol. 27, 4006-4017 (2007)

26. Harley, M. E., Allan, L. A., Sanderson, H. S. \& Clarke, P. R. Phosphorylation of Mcl1 by CDK1-cyclin B1 initiates its Cdc20-dependent destruction during mitotic arrest. EMBO J. 29, 2407-2420 (2010).

27. Inuzuka, H. et al. SCF(FBW7) regulates cellular apoptosis by targeting MCL1 for ubiquitylation and destruction. Nature 471, 104-109 (2011).

28. Magiera, M. M. et al. Trim17-mediated ubiquitination and degradation of Mcl-1 initiate apoptosis in neurons. Cell Death Differ. 20, 281-292 (2013).

29. Zhong, Q., Gao, W., Du, F. \& Wang, X. Mule/ARF-BP1, a BH3-only E3 ubiquitin ligase, catalyzes the polyubiquitination of $\mathrm{Mcl}-1$ and regulates apoptosis. Cell 121, 1085-1095 (2005).

30. Schwickart, M. et al. Deubiquitinase USP9X stabilizes MCL1 and promotes tumour cell survival. Nature 463, 103-107 (2010).

31. Zhang, S. et al. Deubiquitinase USP13 dictates MCL1 stability and sensitivity to BH3 mimetic inhibitors. Nat. Commun. 9, 215 (2018). 